\title{
Refining prognostic stratification of human papillomavirus-related oropharyngeal squamous cell carcinoma: different prognosis between T1 and T2
}

Sumin Lee, MD, Sang-wook Lee, MD, PhD, Sunmin Park, MD, PhD, Sang Min Yoon, MD, PhD, Jin-hong Park, MD, PhD, Si Yeol Song, MD, PhD, Seung Do Ahn, MD, PhD, Jong Hoon Kim, MD, PhD, Eun Kyung Choi, MD, PhD, Su Ssan Kim, MD, PhD, Jinhong Jung, MD, PhD, Young Seok Kim, MD, PhD

Department of Radiation Oncology, Asan Medical Center, University of Ulsan College of Medicine, Seoul, Korea

\begin{abstract}
Purpose: To validate the 8th edition of the American Joint Committee on Cancer/Union for International Cancer Control (AJCC/UICC) TNM staging system for human papillomavirus (HPV)-related oropharyngeal squamous cell carcinoma (OPSCC) and investigate whether a modified classification better reflects the prognosis.

Materials and Methods: Medical records of patients diagnosed with non-metastatic HPV-related OPSCC between 2010 and 2016 at a single institution were retrospectively reviewed. HPV status was determined by immunohistochemical analysis of p16 and/or HPV DNA polymerase chain reaction (PCR). We reclassified TNM stage T0-1 and N0-1 as group A, T2-3 or N2 as B, and T4 or N3 as C. Survival analysis according to 8th AJCC/UICC TNM staging and the modified classification was performed.

Results: Of 383 OPSCC patients, 211 were positive for HPV DNA PCR or p16. After exclusion, 184 patients were included in this analysis. Median age was 56 years (range, 31 to 81 years). Most primary tumors were in the palatine tonsil (148 tumors, 80\%). The eighth AJCC/UICC TNM classification could not differentiate between stage I and II $(p=0.470)$ or II and III $(p=0.209)$. Applying modified grouping, the 3-year overall survival rate of group A was significantly higher than that of group B and C $(98 \%$ vs. $91 \%, p$ $=0.039$ and $98 \%$ vs. $78 \%, p<0.001$, respectively). Differentiation between group $B$ and $C$ was marginally significant $(p=0.053)$.

Conclusion: The 8th AJCC/UICC TNM staging system did not clearly distinguish the prognosis of stage II from that of other stages. Including the T2NO-1 group in stage II may improve prognostic stratification.
\end{abstract}

Keywords: Human papillomavirus, Oropharyngeal neoplasms, Squamous cell carcinoma, Neoplasm staging

\section{Introduction}

The incidence of oropharyngeal squamous cell carcinoma (OPSCC) has been steadily increasing in the United States and Western Europe, accompanied by a rise in the number of patients with human papillomavirus (HPV)-related OPSCC
$[1,2]$. Patients with HPV-related OPSCC tend to be relatively young and less exposed to tobacco smoking and alcohol [3]. HPV-related OPSCC is now well recognized as a distinct entity presenting with more extensive nodal disease but a better prognosis than HPV-unrelated OPSCC [4-6]. As the 7th edition of the American Joint Committee on Cancer/Union

Received 19 September 2017, Revised 26 September 2017, Accepted 26 September 2017.

Correspondence: Sang-wook Lee, MD, PhD, Department of Radiation Oncology, Asan Medical Center, University of Ulsan College of Medicine, 88 Olympic-ro 43-gil, Songpa-gu, Seoul 05505, Korea. Tel: +82-2-3010-4431, Fax: +82-2-3010-6950,

E-mail: Isw@amc.seoul.kr

(c) This is an Open Access article distributed under the terms of the Creative Commons Attribution Non-Commercial License (http://creativecommons.org/ licenses/by-nc/4.0/) which permits unrestricted non-commercial use, distribution, and reproduction in any medium, provided the original work is properly cited.

www.e-roj.org 
for International Cancer Control (AJCC/UICC) TNM staging system for traditional OPSCC does not accurately reflect the prognosis of HPV-related OPSCC, there have been several attempts to propose a new prognostic classification. The International Collaboration on Oropharyngeal cancer Network for Staging (ICON-S) study group proposed a novel prognostic classification for HPV-related OPSCC based on the outcome of over 1,900 patients treated in five North American and two European centers [7], which were adopted by the 8th edition of the AJCC/UICC (8th AJCC/UICC) staging system. Previously, because $\mathrm{N} 1$ to $\mathrm{N} 2 \mathrm{~b}$ stages showed similar overall survival (OS) rates, they were reassigned to $\mathrm{N} 1$, and traditional $\mathrm{N} 2 \mathrm{c}$, bilateral or contralateral nodes, is now N2. The TNM stages were classified as follows according to clinical outcomes: T1-T2 NON1 for stage I, T3 or N2 for stage II, T4 or N3 for stage III, and stage IV reserved for distant metastases.

Because the ICON-S study was based on Western data, it is necessary to evaluate its applicability to patients in other geographic areas. Several Japanese validation studies evaluated the prognostic value of the 8th AJCC/UICC staging system, but somewhat inconsistent results were reported $[8,9]$. Sano et al. [8] could not find any predictive value of the 8th AJCC/UICC staging system, and Mizumachi et al. [9] reported that there was a significant difference in the 3 -year OS rates between stage I-II and III patients, but not between stage I and II. Additionally, applicability of the 8th AJCC/UICC staging system in Korea is still unclear.

Therefore, the first aim of our study was to assess whether the 8th AJCC/UICC staging system could distinguish prognostic groups in patients with HPV-related OPSCC who were treated at a single center in Korea. The second aim was to investigate whether there is a more reflective classification for better prognostic stratification.

\section{Materials and Methods}

\section{Patients}

We retrospectively reviewed the medical records of patients diagnosed with non-metastatic OPSCC between January 2010 and December 2016 at our institution. All patients were staged according to the 8th AJCC/UICC TNM staging system using physical and endoscopic exams, computed tomography (CT), magnetic resonance imaging (MRI), and 18F-fluorodeoxyglucose positron emission tomography (PET)/ CT imaging. Patients with a history of another malignancy, radiotherapy (RT) for head and neck lesions, or chemotherapy were excluded from this analysis. The protocol was reviewed and approved by the Institutional Review Board of Asan Medical Center, Korea. Identifying number is 2017-1106.

\section{Evaluation of HPV status}

Two methods are mainly used to evaluate HPV infection status in our institution. One is tumor p16 immunohistochemical (IHC) analysis using CINtec p16 histology (anti-p16 ${ }^{\text {INK4a }}$ mouse monoclonal antibody and IHC detection kit; Roche MTM Laboratories, Heidelberg, Germany). A positive p16 result was defined as diffuse strong staining in the cytoplasm and nucleus. Focal or faint reactivity was considered negative for p16. The other method is tumor HPV DNA detection by polymerase chain reaction (PCR)/DNA chip scanning, which can detect 43 subtypes of HPV (high-risk subtypes of 16, 18, $31,33,35,39,45,51,52,56,58,59,68,73,82$, and other lower or undetermined risk subtypes). HPV-related OPSCC was diagnosed when either p16 or HPV DNA PCR results were positive.

\section{Treatment and follow-up}

Patients received either upfront surgery or definitive chemoradiotherapy. Initial decision to perform surgery was made by a surgeon. As a radical surgery, wide excision of the primary tumor with modified radical or selective neck dissection of the lymph nodes on the involved or suspected side of the neck was performed. Postoperative radiotherapy was recommended for patients who had adverse feature in pathologic report: positive surgical resection margin, pathologic T3, or node positive disease. Adjuvant chemotherapy was performed when an advanced nodal stage or extracapsular extension (ECE) was identified in pathologic reports. Concurrent chemoradiotherapy included cisplatinbased (100 mg/m $\mathrm{m}^{2}$ every 3 weeks) chemotherapy. RT was delivered 5 days per week with intensity-modulated RT or volumetric modulated arc therapy. A total dose of RT was approximately 60-65 Gy at 1.8-2.0 Gy per fraction in the postoperative setting and approximately $70 \mathrm{~Gy}$ at $2.0-2.2 \mathrm{~Gy}$ per fraction in the definitive setting.

After treatment, the first follow-up was conducted a month after definitive RT or within 6 months after surgery using $\mathrm{CT}$, $\mathrm{MRI}$, and/or PET/CT. Patients were then followed up at least twice a year for physical examinations and at least once a year for imaging.

\section{Statistical analysis}

Chi-square tests were used to compare categorical variables. The primary endpoint was OS, which was calculated from 
the date of diagnosis to either death from any cause or the last date on which the patient was known to be alive. The Kaplan-Meier method and Wilcoxon log-rank test were used for survival analysis. For univariate and multivariate analysis, Cox proportional hazards models were used. The hazard ratios, 95\% confidence intervals, and $p$-values were calculated. A $p$-value of $<0.05$ was considered statistically significant. SPSS ver. 22 (IBM SPSS, Armonk, NY, USA) was used for all statistical analyses.

\section{Results}

\section{Patient characteristics}

Between January 2010 and December 2016, 383 patients with OPSCC were diagnosed at our institution. Of these, p16 testing or HPV DNA PCR was performed in 264 patients, and 211 were identified as having HPV-related OPSCC. Twenty-seven patients were excluded: 18 who were not followed up at our institution, 3 who had initially metastatic or recurrent disease, and 6 who had a history of another malignancy. Finally, 184 patients were included in this analysis and their characteristics are shown in Table 1 . The median age of the patients was 56 years (range, 31 to 81 years), and most patients were men (84\%). Fiftyeight patients $(32 \%)$ had a smoking history of over 20 packyears. The palatine tonsil was the most common primary site (148 patients, 80\%), and there were $22(12 \%)$ cancers of the base of the tongue, 2 (1\%) soft palate cancers, and 12 (7\%) metastatic neck lymph nodes with an unknown primary site. The proportions of moderately and poorly differentiated squamous cell carcinoma were similar. The HPV-related OPSCC was stage I in 134 (73\%), stage II in 23 (13\%), and stage III in 27 (15\%) patients based on the 8th AJCC/UICC staging system. Upfront radical surgery was performed in 112 patients (61\%).

\section{The 8th AJCC/UICC staging and survival}

The median follow-up time was 38 months (range, 5 to 88 months). The 3-year and 5-year OS rates for all patients were 91.3\% and 86.5\%, respectively. In the T category of the 8th AJCC/UICC staging, relatively good prognostic stratification was observed (overall $p=0.024$ ) (Fig. 1A). T4 disease showed a worse survival than $\mathrm{T} 1(p=0.002)$ and there was a marginally significant difference between $\mathrm{T} 1$ and $\mathrm{T} 2$ disease $(p=0.085)$ and between T2 and T4 disease ( $p=0.060)$. After adjusted by age, T4 disease was still showed a significantly worse survival than T1 and T2 disease ( $p=0.009$ and $p=0.039$, respectively). On the contrary, the $\mathrm{N}$ category of the 8th AJCC/UICC staging could not distinguish the prognoses in NO through N3 disease
Table 1. Patient characteristics $(n=184)$

\begin{tabular}{|c|c|}
\hline Characteristic & Value \\
\hline Age (yr) & $56(31-81)$ \\
\hline \multicolumn{2}{|l|}{ Sex } \\
\hline Male & $154(84)$ \\
\hline Female & $30(16)$ \\
\hline \multicolumn{2}{|l|}{ ECOG performance status } \\
\hline $0-1$ & $179(97)$ \\
\hline $2-3$ & $5(3)$ \\
\hline \multicolumn{2}{|l|}{ Alcohol (standard drinks/week) a) } \\
\hline$\leq 10$ & $148(80)$ \\
\hline$>10$ & $36(20)$ \\
\hline \multicolumn{2}{|l|}{ Smoking pack-years } \\
\hline Never smoker & $67(36)$ \\
\hline$\leq 10$ & $31(17)$ \\
\hline$\leq 20$ & $28(15)$ \\
\hline$>20$ & $58(32)$ \\
\hline \multicolumn{2}{|l|}{ Primary site } \\
\hline Palatine tonsil & $148(80)$ \\
\hline Base of tongue & $22(12)$ \\
\hline Soft palate & $2(1)$ \\
\hline Metastatic neck lymph nodes with unknown primary & $12(7)$ \\
\hline \multicolumn{2}{|c|}{ Histology } \\
\hline SCC, WD & $2(1)$ \\
\hline SCC, MD & $98(53)$ \\
\hline SCC, PD & $81(44)$ \\
\hline Others & $3(2)$ \\
\hline \multicolumn{2}{|l|}{ Clinical T-stage (8th AJCC/UICC) } \\
\hline 0 & $12(7)$ \\
\hline 1 & $53(29)$ \\
\hline 2 & $88(48)$ \\
\hline 3 & $9(5)$ \\
\hline 4 & $22(12)$ \\
\hline \multicolumn{2}{|l|}{ Clinical N-stage (8th AJCC/UICC) } \\
\hline 0 & $23(13)$ \\
\hline 1 & $131(71)$ \\
\hline 2 & $25(14)$ \\
\hline 3 & $5(3)$ \\
\hline \multicolumn{2}{|l|}{ Stage (8th AJCC/UICC) } \\
\hline I & $134(73)$ \\
\hline II & $23(13)$ \\
\hline III & $27(15)$ \\
\hline \multicolumn{2}{|l|}{ Primary treatment } \\
\hline Surgery & $112(61)$ \\
\hline Radiation & 72 (39) \\
\hline
\end{tabular}

Values are presented as median (range) or number (\%). ECOG, Eastern Cooperative Oncology Group; SCC, squamous cell carcinoma; $W D$, well differentiated; $M D$, moderately differentiated; PD, poorly differentiated; 8th AJCC/UICC, American Joint Committee on Cancer/Union for International Cancer Control staging 8th edition.

a) A standard drink is any drink that contains approximately $14 \mathrm{~g}$ of pure alcohol. 
A

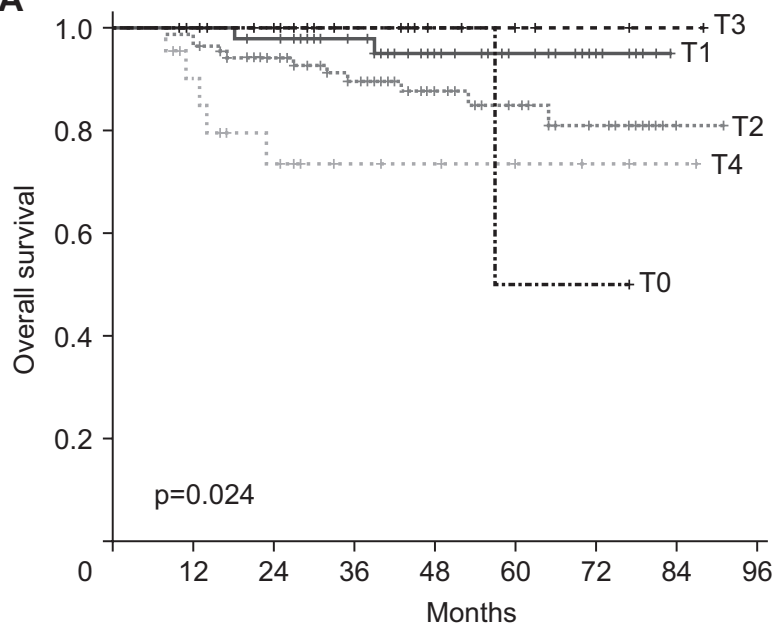

$\begin{array}{lcccccccc}\text { No. at risk } \\ \text { T0 } & 12 & 11 & 7 & 6 & 3 & 1 & 1 & 0 \\ \text { T1 } & 53 & 51 & 45 & 37 & 24 & 16 & 8 & 0 \\ \text { T2 } & 88 & 84 & 68 & 55 & 37 & 27 & 16 & 1 \\ \text { T3 } & 9 & 9 & 9 & 5 & 4 & 3 & 2 & 1 \\ \text { T4 } & 22 & 17 & 12 & 6 & 5 & 3 & 2 & 1\end{array}$

C

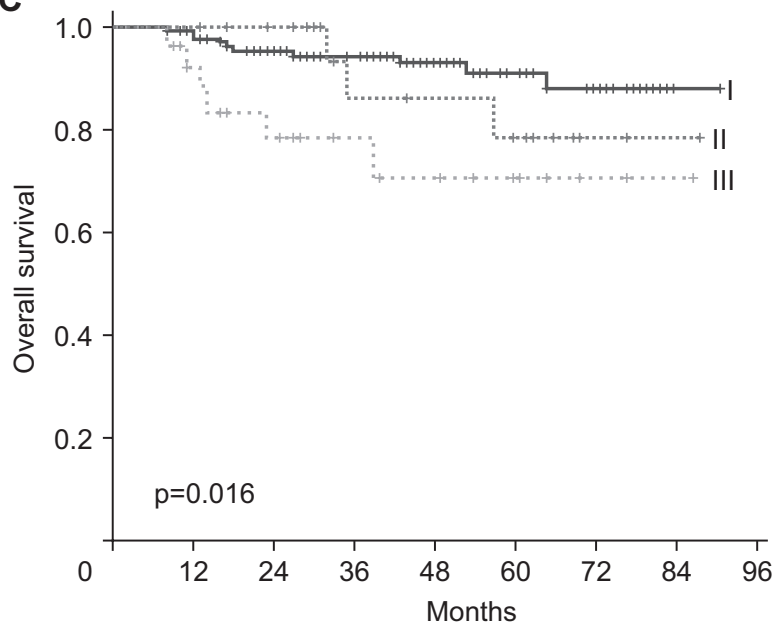

No. at risk

$\begin{array}{lllllllll}\text { I } & 134 & 128 & 106 & 87 & 54 & 36 & 24 & 1\end{array}$

$\begin{array}{lllllllll}\text { II } & 23 & 23 & 19 & 12 & 11 & 9 & 3 & 1\end{array}$

$\begin{array}{lllllllll}\text { III } & 27 & 21 & 16 & 10 & 8 & 5 & 2 & 1\end{array}$

(overall $p=0.243$ ) (Fig. 1B). The 3-year OS rates for patients with stage I, II, and III disease were $94.4 \%, 86.2 \%$, and $78.4 \%$, respectively (Fig. 1C). There was a significant difference in OS between stage I and III ( $p=0.005)$, but stage II was not significantly different from stage I or III $(p=0.470$ and $p=$ 0.209, respectively).

Univariate and multivariate analyses of prognostic factors for OS were performed (Table 2). Age $>56$ years and the 8th AJCC/UICC stage were the only significant prognostic factors in
B

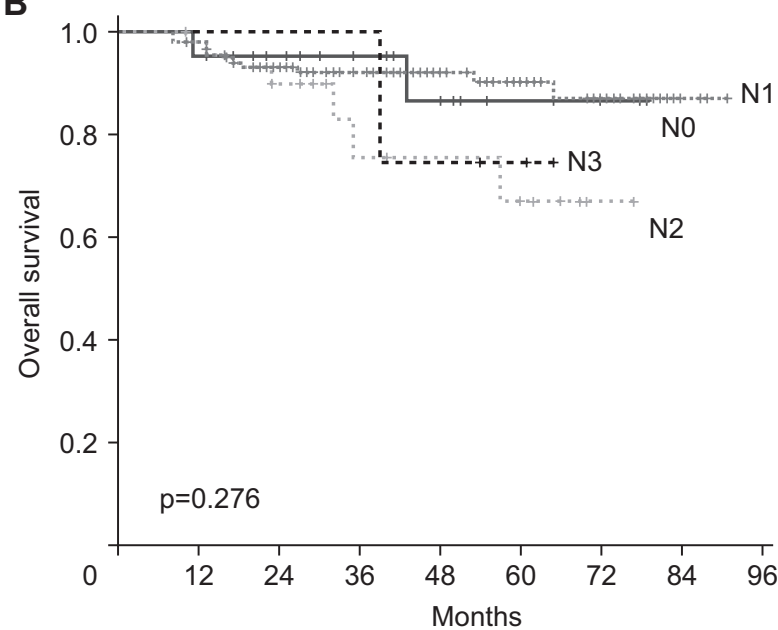

No. at risk

$\begin{array}{ccccccccc}\text { N0 } & 23 & 22 & 18 & 13 & 9 & 6 & 3 & 0 \\ \text { N1 } & 131 & 123 & 103 & 82 & 52 & 35 & 24 & 3 \\ \text { N2 } & 25 & 23 & 16 & 10 & 9 & 7 & 2 & 0 \\ \text { N3 } & 5 & 4 & 4 & 4 & 3 & 2 & 0 & 0\end{array}$

Fig. 1. Kaplan-Meier curves for overall survival for patients according to $T$ category (A), N category (B), and the TNM classification (C) using the 8th edition of the American Joint Committee on Cancer/Union for International Cancer Control (AJCC/UICC) TNM staging system.

univariate analysis ( $p=0.044$ and $p=0.027$, respectively), the prognostic impact of which was retained in the multivariate analysis ( $p=0.022$ and $p=0.011$, respectively). Other clinical factors including smoking and alcohol history, primary site, histology, ECE, primary treatment, and chemotherapy were not significant prognostic factors for $\mathrm{OS}$.

In a subgroup analysis of patients aged $\leq 56$ or $>56$ years, there was no difference in OS according to the 8th AJCC/UICC staging in the younger patients of 56 years or less (overall 
Table 2. Comparison of overall survival by clinical factors

\begin{tabular}{|c|c|c|c|c|}
\hline \multirow{2}{*}{ Variable } & \multicolumn{2}{|c|}{ Univariate analysis } & \multicolumn{2}{|c|}{ Multivariate analysis } \\
\hline & $\mathrm{HR}(95 \% \mathrm{Cl})$ & p-value & $\mathrm{HR}(95 \% \mathrm{Cl})$ & $p$-value \\
\hline \multicolumn{5}{|l|}{ Age (yr) } \\
\hline$\leq 56(n=95)$ & Reference & & Reference & \\
\hline$>56(n=89)$ & $2.712(1.028-7.150)$ & $0.044^{*}$ & $3.183(1.186-8.542)$ & $0.022^{*}$ \\
\hline \multicolumn{5}{|l|}{ Sex } \\
\hline Male $(n=154)$ & Reference & & & \\
\hline Female $(n=30)$ & $0.572(0.132-2.478)$ & 0.455 & & \\
\hline \multicolumn{5}{|c|}{ Alcohol (standard drinks/week) } \\
\hline$\leq 10(n=148)$ & Reference & & & \\
\hline$>10(n=36)$ & $1.005(0.333-3.030)$ & 0.993 & & \\
\hline \multicolumn{5}{|l|}{ Smoking pack-years } \\
\hline$\leq 10(n=98)$ & Reference & & & \\
\hline$>10(n=86)$ & $1.523(0.612-3.788)$ & 0.365 & & \\
\hline Subsite & & 0.947 & & \\
\hline Palatine tonsil $(n=148)$ & Reference & & & \\
\hline Base of tongue $(n=22)$ & $1.461(0.423-5.051)$ & 0.549 & & \\
\hline Soft palate $(n=2)$ & 0.000 & 0.984 & & \\
\hline Neck lymph nodes $(n=12)$ & $0.987(0.130-7.491)$ & 0.990 & & \\
\hline Histology & & 0.492 & & \\
\hline$S C C, W D(n=2)$ & $5.080(0.638-40.438)$ & 0.125 & & \\
\hline SCC, MD (n = 98) & Reference & & & \\
\hline $\mathrm{SCC}, \mathrm{PD}(\mathrm{n}=81)$ & $1.061(0.421-2.677)$ & 0.900 & & \\
\hline Others $(n=3)$ & 0.000 & 0.982 & & \\
\hline \multicolumn{5}{|l|}{ Clinical ECE } \\
\hline No $(n=155)$ & Reference & & & \\
\hline Yes $(n=29)$ & $1.331(0.441-4.011)$ & 0.612 & & \\
\hline \multicolumn{5}{|l|}{ Primary treatment } \\
\hline Surgery $(n=112)$ & Reference & & & \\
\hline Radiation therapy $(n=72)$ & $0.758(0.308-1.869)$ & 0.548 & & \\
\hline \multicolumn{5}{|l|}{ Chemotherapy } \\
\hline No $(n=78)$ & Reference & & & \\
\hline Yes $(n=106)$ & $0.828(0.332-2.069)$ & 0.687 & & \\
\hline Stage (8th AJCC/UICC) & & $0.027^{*}$ & & $0.011^{*}$ \\
\hline $\mid(n=134)$ & Reference & & Reference & \\
\hline$\|(n=23)$ & $1.651(0.453-6.014)$ & 0.447 & $1.596(0.438-5.812)$ & 0.479 \\
\hline III $(\mathrm{n}=27)$ & $4.017(1.456-11.083)$ & $0.007^{*}$ & $4.860(1.729-13.665)$ & $0.003^{*}$ \\
\hline
\end{tabular}

$\mathrm{HR}$, hazard ratio; $\mathrm{Cl}$, confidence interval; SCC, squamous cell carcinoma; WD, well differentiated; MD, moderately differentiated; $\mathrm{PD}$, poorly differentiated; ECE, extracapsular extension; 8th AJCC/UICC, American Joint Committee on Cancer/Union for International Cancer Control staging 8th edition.

${ }^{a)} \mathrm{A}$ standard drink is any drink that contains approximately $14 \mathrm{~g}$ of pure alcohol.

${ }^{*} p<0.05$.

$p=0.313)$. The difference in OS between stage I and III was observed in patients older than 56 years ( $p=0.003$ ), stage II still was not significantly different from stage I or III in older age group ( $p=0.155$ and $p=0.133$, respectively).

\section{Refining prognostic classification}

Because intersection of survival curves of stage I and II was observed and more patients were assigned to stage I than stage II and III, we tried to divide stage I into the following two groups: T0-1 N0-1 and T2 N0-1. This separation showed that the T2 N0-1 and stage II patients had similar survival rates (Fig. 2A). Accordingly, we reclassified the prognostic group of the 8th AJCC/UICC staging as follows: T0- 1 N0-1 as group $A$, T2-3 or N2 as group B, and T4 or N3 as group C (Fig. 3). The 3 -year OS rates of refined groups $A, B$, and $C$ were $98 \%, 91 \%$, and $78 \%$, respectively, and this refined staging showed better 
A

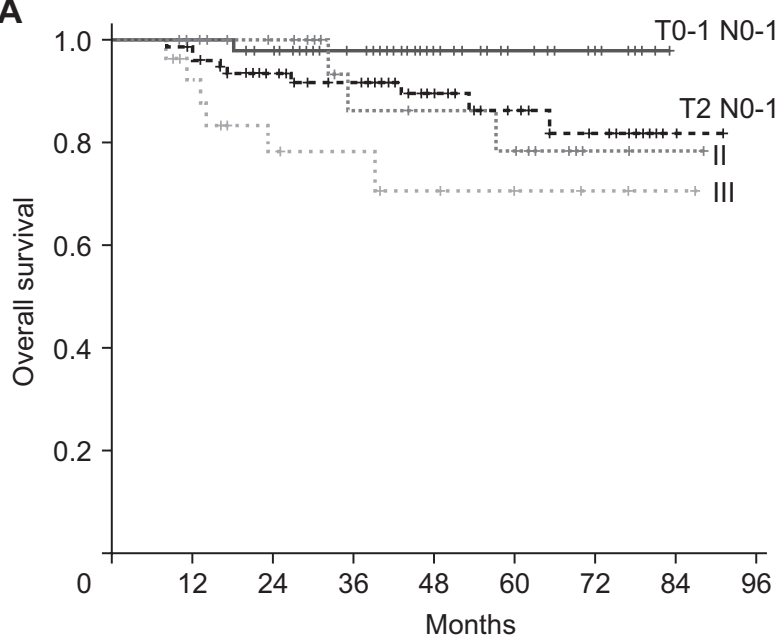

No. at risk

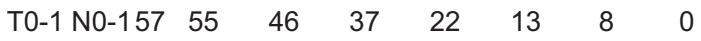

T2 N0-1 $77 \begin{array}{lllllll}73 & 60 & 50 & 32 & 23 & 16 & 0\end{array}$

II $\quad \begin{array}{llllllll}23 & 23 & 19 & 12 & 11 & 9 & 3 & 1\end{array}$

III $\quad \begin{array}{llllllll}27 & 21 & 16 & 10 & 8 & 5 & 2 & 1\end{array}$
B

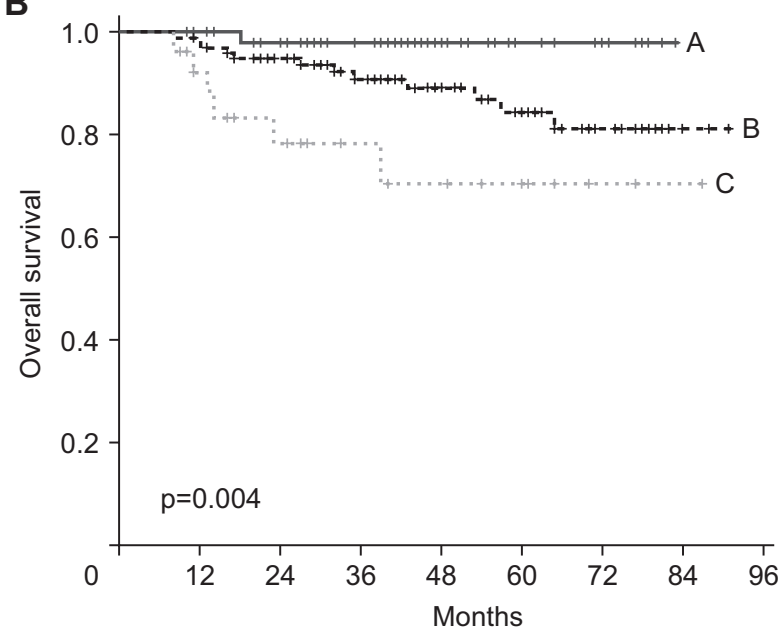

No. at risk

$\begin{array}{ccccccccc}\text { A } & 57 & 55 & 46 & 37 & 22 & 13 & 8 & 0 \\ \text { B } & 100 & 96 & 79 & 62 & 43 & 32 & 19 & 2 \\ \text { C } & 27 & 21 & 16 & 10 & 8 & 5 & 2 & 1\end{array}$

Fig. 2. Kaplan-Meier curves of TO-1 NO-1 group and T2 N0-1 group, stage II and III using the 8th edition of American Joint Committee on Cancer/Union for International Cancer Control (AJCC/UICC) TNM staging system (A) and refined staging (B): T0-1 N0-1 as group A, T2-3 or N2 as group B, and T4 or N3 as group C.

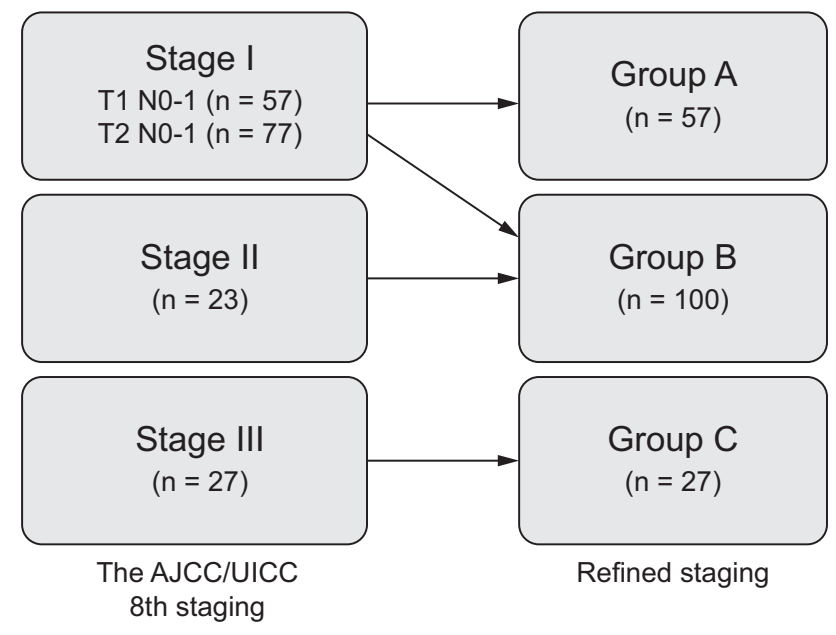

Fig. 3. The shift in stage among patients with HPV-related OPSCC from the 8th edition of the American Joint Committee on Cancer/ Union for International Cancer Control (AJCC/UICC) TNM staging to refined staging: T0-1 N0-1 as group A, T2-3 or N2 as group $\mathrm{B}$, and $\mathrm{T} 4$ or N3 as group $\mathrm{C}$.

prognostic stratification than the 8th AJCC/UICC staging (overall $p=0.004$ ) (Fig. 2B).

In a multivariate analysis for $O S$ that included refined stage instead of the 8th AJCC/UICC stage, refined stage was still an independent prognostic factor (overall $p=0.009$ ), although the distinction of the adjusted hazard ratio between groups $A$ and $B$ became statistically less significant (adjusted hazard ratio for group $B$ to group $A=5.386 ; p=0.107$ )

In a subgroup analysis, there was no difference in OS according to the refined staging in the younger patients under 56 years of age (overall $p=0.320$ ) (Fig. $4 \mathrm{~A}$ ), but the difference in OS according to the refined group became clear in patients over 56 years of age (overall $p=0.003$ ) (Fig. 4B). In older age group, statistical significance of the difference in OS between Group B and other groups was higher than the 8th AJCC/UICC staging ( $p$ $=0.081$ for group $B$ vs. $A ; p=0.012$ for group $B$ vs. $C$ ).

\section{Discussion and Conclusion}

As previously reported in several studies $[4,10,11]$, the present study also confirmed a favorable outcome of HPV-related OPSCC, reporting 3-year and 5-year OS rates for all patients of $91.3 \%$ and $86.5 \%$, respectively. Nodal status is known to have less influence on prognosis despite a relatively extensive nodal spread at presentation. Although the ICON-S study group distinguished between NO and N1 (ipsilateral lymph node involvement), because there was no difference in survival between the two groups, they were classified into the same prognostic group. The present study also did not find a difference in survival between $\mathrm{NO}$ and $\mathrm{N} 1$ groups. In 
A

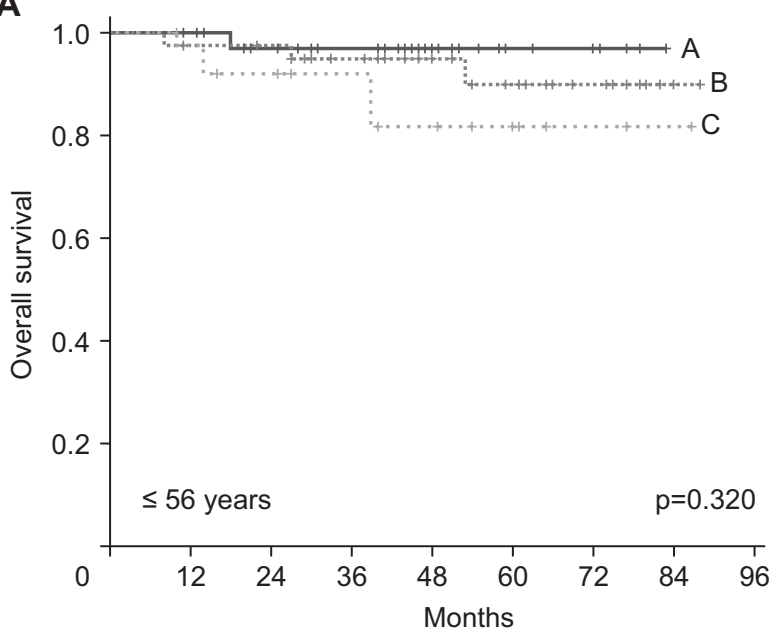

No. at risk

$\begin{array}{ccccccccc}\text { A } & 39 & 37 & 31 & 25 & 15 & 8 & 5 & 0 \\ \text { B } & 42 & 40 & 38 & 32 & 22 & 17 & 10 & 0 \\ \text { C } & 14 & 13 & 11 & 9 & 7 & 4 & 2 & 1\end{array}$

B

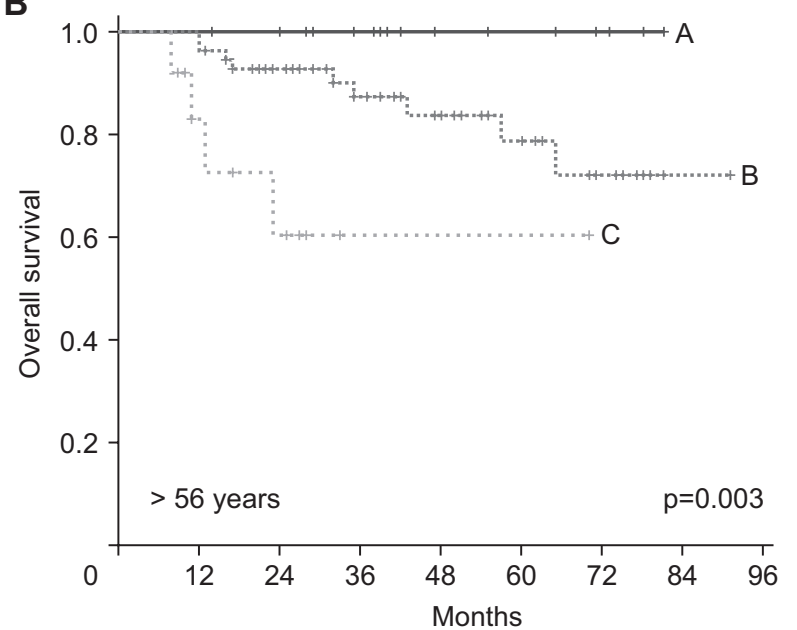

No. at risk

$\begin{array}{ccccccccc}\text { A } & 18 & 18 & 15 & 12 & 7 & 5 & 3 & 0 \\ \text { B } & 58 & 56 & 41 & 30 & 21 & 15 & 9 & 1 \\ \text { C } & 13 & 8 & 5 & 1 & 1 & 1 & 0 & 0\end{array}$

Fig. 4. Kaplan-Meier curves of refined staging for patients $\leq 56$ years (A) and $>56$ years (B).

contrast, the T category showed a relatively better prognostic stratification in this case series.

In the present study, stage II did not show a significant survival difference from stage I or III. Similar results were obtained in a validation study conducted in Japan [9]. The authors evaluated 111 patients with p16 positive OPSCC treated at a single institution in Japan. The 3-year OS rate of stage III was $70.2 \%$, which is significantly lower than stage I and II. But, the 3-year OS rates of stage I and II were the same at $90.9 \%$. The ICON-S study group also showed excellent prognostic stratification according to the 8th AJCC/ UICC staging in both training and validation cohort. But the survival curve of stage II was located closer to that of stage I than stage III, with a relatively small difference between the survival curves of stage I and II compared with the difference between those of stages II and III. These similar results suggest that there is room for increasing the prognostic value of TNM classification by modifying the definition of stage II.

In HPV-related OPSCC, the T stages were more influential in the survival outcome than the $\mathrm{N}$ stages. Therefore, dividing stage I into T1 and T2 groups is considered a reasonable approach. After we classified the T2 N0-1 group as stage II, the survival analysis with the refined grouping showed a clearer separation of stage II from the other stages.

Our univariate and multivariate analyses revealed that age $\leq 56$ years is the only independent prognostic factor except
TNM classification. The fact that age is one of the important prognostic factors has also been shown in previous studies evaluating the clinical outcome of HPV-related OPSCC $[4,7,8]$. The present study shows that the difference in survival rate according to refined staging is mainly observed in patients older than 56 years of age. In patients younger than 56 years, there are no significant differences in survival rates between stages I, II, and even III. Younger age may act as an independent prognostic factor, diluting the difference in survival rates due to stage. We could not find definite evidence of any difference in patient- or treatment-related factors that affect survival between young and old patient groups. Despite inferior survival in elderly patients, Dave et al. [12] reported a favorable survival outcome for patients older than 65 years with a high tolerability for treatment and insisted that elderly patients should be considered for stage-appropriate care.

Clinical factors other than age did not significantly affect the prognosis of HPV-related OPSCC. The 8th AJCC/UICC TNM staging system introduced the use of ECE in the N category but not for HPV-related OPSCC. Several conflicting results have been reported on prognostic impact of ECE [1315]. In the present study, we could not find any prognostic value of ECE. Because determination of ECE was based on radiologic findings, accuracy would be reduced. Also, biased result might be derived because pathologically confirmed ECE was indication of concurrent chemotherapy in postoperative 
setting. However, considering that the degree of lymph node metastasis in the HPV-related OPSCC has a relatively small impact on prognosis, ECE might also be less influential on survival than other malignancies. Further studies on the impact of ECE on prognosis are warranted.

The limitations of this study include its retrospective nature based in a single institution and a lack of treatment standardization. Furthermore, unlike the TNM/UICC staging system using immunohistochemistry for p16 overexpression, p16 and HPV DNA tests were used to classify HPV-mediated OPSCC in the present study. There was only one case of p16positive/HPV DNA-negative OPSCC, but 23 patients had $p 16$ negative/HPV DNA-positive OPSCC. It is not yet clear whether the prognosis of patients with these inconsistent results for p16 and HPV DNA will differ.

In conclusion, stage II in the 8th AJCC/UICC TNM staging system did not clearly distinguish the prognosis from other stages, and inclusion of the T2N0-1 group in stage II instead of stage I could improve prognostic stratification. Age was independent prognostic factor for HPV-related OPSCC and survival differences according to refined stage are mainly present in older patients.

\section{Conflict of Interest}

No potential conflict of interest relevant to this article was reported.

\section{References}

1. Marur S, D'Souza G, Westra WH, Forastiere AA. HPV-associated head and neck cancer: a virus-related cancer epidemic. Lancet Oncol 2010;11:781-9.

2. Chaturvedi AK, Engels EA, Pfeiffer RM, et al. Human papillomavirus and rising oropharyngeal cancer incidence in the United States. J Clin Oncol 2011;29:4294-301.

3. Chaturvedi AK, Engels EA, Anderson WF, Gillison ML. Incidence trends for human papillomavirus-related and -unrelated oral squamous cell carcinomas in the United States. J Clin Oncol 2008;26:612-9.

4. Ang KK, Harris J, Wheeler R, et al. Human papillomavirus and survival of patients with oropharyngeal cancer. N Engl J Med 2010;363:24-35.
5. Hafkamp HC, Manni JJ, Haesevoets A, et al. Marked differences in survival rate between smokers and nonsmokers with HPV 16-associated tonsillar carcinomas. Int J Cancer 2008;122:2656-64.

6. Ragin CC, Taioli E. Survival of squamous cell carcinoma of the head and neck in relation to human papillomavirus infection review and meta-analysis. Int J Cancer 2007;121:1813-20.

7. O'Sullivan B, Huang SH, Su J, et al. Development and validation of a staging system for HPV-related oropharyngea cancer by the International Collaboration on Oropharyngeal cancer Network for Staging (ICON-S): a multicentre cohort study. Lancet Oncol 2016;17:440-51.

8. Sano D, Yabuki $K$, Arai Y, et al. The applicability of new TNM classification for humanpapilloma virus-related oropharyngeal cancer in the 8th edition of the AJCC/UICC TNM staging system in Japan: a single-centre study. Auris Nasus Larynx 2017 Jul 28 [Epub]. http://doi.org/10.1016/j.anl.2017.07.010.

9. Mizumachi T, Homma A, Sakashita T, Kano S, Hatakeyama $H_{\text {, }}$ Fukuda S. Confirmation of the eighth edition of the AJCC/UICC TNM staging system for HPV-mediated oropharyngeal cancer in Japan. Int J Clin Oncol 2017;22:682-9.

10. Fakhry C, Zhang Q, Nguyen-Tan PF, et al. Human papillomavirus and overall survival after progression of oropharyngeal squamous cell carcinoma. J Clin Oncol 2014;32:3365-73.

11. Keane FK, Chen $Y H$, Neville BA, et al. Changing prognostic significance of tumor stage and nodal stage in patients with squamous cell carcinoma of the oropharynx in the human papillomavirus era. Cancer 2015;121:2594-602.

12. Dave E, Su W, Gupta V, et al. Human Papilloma Virus-positive Oropharyngeal Squamous Cell Carcinoma in the Elderly. Anticancer Res 2017;37:1847-51.

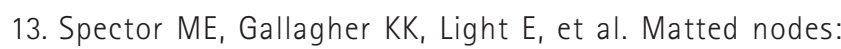
poor prognostic marker in oropharyngeal squamous cell carcinoma independent of HPV and EGFR status. Head Neck 2012;34:1727-33.

14. An Y, Park HS, Kelly JR, et al. The prognostic value of extranodal extension in human papillomavirus-associated oropharyngeal squamous cell carcinoma. Cancer 2017;123:2762-72.

15. Sinha P, Lewis JS Jr, Piccirillo JF, Kallogjeri D, Haughey $\mathrm{BH}$. Extracapsular spread and adjuvant therapy in human papillomavirus-related, p16-positive oropharyngeal carcinoma. Cancer 2012;118:3519-30. 